\title{
Strange metals at finite 't Hooft coupling
}

\author{
Kazem Bitaghsir Fadafan ${ }^{1}$ \\ ${ }^{2}$ Physics Department, Shahrood University of Technology, \\ P.O.Box 3619995161, Shahrood, Iran \\ E-mails: bitaghsir@shahroodut.ac.ir
}

\begin{abstract}
In this paper, we consider the AdS-Schwarzshild black hole in light-cone coordinates which exhibits non-relativistic $\mathrm{z}=2$ Schrodinger symmetry. Then, we use the $A d S / C F T$ correspondence to investigate the effect of finite-coupling corrections to two important properties of the strange metals which are the Ohmic resistivity and the inverse Hall angle. It is shown that the Ohmic resistivity and inverse Hall angle are linear and quadratic temperature dependent in the case of $\mathcal{R}^{4}$ corrections, respectively. While in the case of Gauss-Bonnet gravity, we find that the inverse Hall angle is quadratic temperature dependent and the Ohmic conductivity can never be linear temperature dependent.
\end{abstract}




\section{Contents}

1 Introduction

2 Non-relativistic DC conductivity from AdS Light-Cone black hole 3

3 Strange metals from AdS Light-Cone black hole 5

3.1 The resistivity of strange metals at finite coupling . . . . . . . . 8

3.2 Hall conductivity of strange metals at finite coupling . . . . . . . . 12

4 Conclusion

13

\section{Introduction}

The AdS/CFT correspondence has been a powerful tool for studying dynamics of strongly coupled field theory. Recently the application of this duality in condensed matter physics (called $A d S / C M T$ ) has been studied [1]. One of the most interesting subjects in this content is to understand the strange metal behavior of heavy fermion compounds and high temperature superconductors [2, 3. They show two important properties: linear temperature dependent resistivity and quadratic temperature dependent inverse Hall angle which can be expressed as

$$
\rho=\left(\sigma^{y y}\right)^{-1} \sim T, \quad\left(\cot \theta_{H}\right)^{-1} \sim \frac{\sigma^{y y}}{\sigma^{y z}} \sim T^{2} .
$$

Because of the Hall current due to the magnetic field, $\theta_{H}$ is called the Hall angle. The other property of strange metals is related to the scaling of $\mathrm{AC}$ conductivity which we will not pursuit in this paper. It is widely believed that to gain a better understanding of such properties requires us to go beyond the regime of weak coupling [4].

To study non-relativistic phenomena in condensed matter, one should investigate the non-relativistic generalization of the $A d S / C F T$ correspondence which has become an active research area [5, 6, 7]. By introducing the Schrödinger symmetry the Schrödinger space successfully fitted into the AdS/CFT correspondence. A direct method to obtain this space is to use the Null Melvin Twist to the known solutions of type II supergravity [8]. Another way is to use the light-cone coordinates, then Schrödinger space can be found in the pure $A d S$ [9]. Properties of Schrodinger black holes from those of AdS black holes expressed in light-cone coordinates were discussed in [10].

Using the AdS/CFT, The strange metal behaviors were studied in 11, 12, 13, 14, 15, 16. 17. . One should notice that there exists a few holographic systems which show the particular temperature dependence of the Ohmic resistivity and Hall angle in (1). One also finds that the gravitational solutions showing the Lifshitz-like scaling does not reproduce the behavior of the Hall angle [18. A holographic model building approach to strange metallic phenomenology was proposed in [14] and it was argued that the spatial part of the metric components of background metric should not be same.

In our study, we will consider $A d S$ space in the light-cone frame (ALCF). This frame is proposed as a physical system which describes the physics of strange metals [19]. Comparing ALCF background and a Lifshitz background was discussed in [20]. It was shown that how an extra parameter can change the temperature scaling behavior of conductivity. 
Our purpose is to find the temperature dependence of (11) in the presence of higher derivative corrections. These corrections on the gravity side correspond to finite-coupling corrections on the gauge theory side. The main motivation to consider corrections comes from the fact that string theory contains higher derivative corrections arising from stringy effects. In the case of $\mathcal{N}=4 \mathrm{SYM}$ theory, the leading order correction in $1 / \lambda$ arises from stringy correction to the low energy effective action of type $\amalg b$ supergravity, $\alpha^{\prime 3} \mathcal{R}^{4}$. The DC conductivity of massive $\mathcal{N}=2$ hypermultiplet fields in an $\mathcal{N}=4 S U\left(N_{c}\right)$ super-YangMills theory plasma in the large $N_{c}$ and finite 't Hooft coupling was studied in [21]. Here, we continue this study to investigate $\mathcal{R}^{4}$ and Gauss-Bonnet corrections to two important properties of strange metals in (11). An understanding of how the strange metal behaviors affected by finite $\lambda$ corrections may be essential for theoretical predictions.

The article is organized as follows. In the next section, we will present the basic idea for studying the temperature dependence of the resistivity in the ALCF framework. We follow the same direction and consider the corrections to the strange metal behaviors in section 3 . In the last section we draw our conclusions and summarize our results.

\section{Non-relativistic DC conductivity from AdS Light-Cone black hole}

In this section, we explore calculating the non-relativistic DC conductivity in ALCF. The non-relativistic DC conductivity from the Schrodinger black hole spacetime has been obtained in 22]. They use the dual gravitational description of probe D7 branes in an asymptotically Schrodinger spacetime.

We consider the full 10-dimensional spacetime as $A d S_{5}$ times $S_{5}$ metric

$$
d s^{2}=G_{t t} d t^{2}+G_{x x} d x^{2}+G_{y y} d y^{2}+G_{z z} d z^{2}+G_{u u} d u^{2}+d^{2} \Omega_{5} .
$$

Here $(x, y, z, t)$ are field theory space and the metric functions are given by $G_{t t}, G_{x x}, G_{y y}, G_{z z}$ and $G_{u u}$. The radial coordinates denotes by $u$. The $A d S$ boundary is located at infinity and $u_{0}$ is event horizon. We will drop the $S_{5}$ part of the metric for the rest of our discussion and discuss it when introduce D7 branes on the background (2) 1

The metric of $A L C F$ is obtained from (2) by the transformation $x^{+}=b(t+x)$ and $x^{-}=\frac{1}{2 b}(t-x)$, which yields [10, 22]

$$
\begin{gathered}
d s^{2}=g_{++} d x^{+2}+2 g_{+-} d x^{+} d x^{-}+g_{--} d x^{-2}+ \\
G_{y y} d y^{2}+G_{z z} d z^{2}+G_{u u} d u^{2}
\end{gathered}
$$

where

$$
g_{++}=\frac{G_{t t}+G_{x x}}{4 b^{2}}, \quad g_{+-}=\frac{G_{t t}-G_{x x}}{2}, g_{--}=b^{2}\left(G_{t t}+G_{x x}\right) .
$$

This coordinate transformation is introduced in [7].

The light-cone direction $x^{+}$is identified as time and the momentum of the $x^{-}$direction is fixed. The symmetry is broken to the Lifshitz $(z=2)$ symmetry. A key ingredient of

\footnotetext{
${ }^{1}$ One should notice that in the Schrodinger spacetime this part of the metric is very important becasue of the non-trivial Kalb-Ramond field 22 .
} 
ALCF is transition from AdS $(z=1)$ to Lifshitz $(z=2)$. This transition is controlled by the parameter $b$. In brief, the corresponding dual theory interpolates between the usual relativistic scale symmetry and the Lifshitz symmetry. The role and interpretation of the parameter $b$ is explored in [19]. One finds that thermodynamic properties of the ALCF are identical to those of Schrödinger space [10].

One should introduce $N_{f}$ D7 branes on the background metric of ALCF (3) to calculate the non-relativistic DC conductivity. Notice that we work in the probe limit so that $N_{f}<<$ $N_{c}$. Then we ignore the quantum effects due to flavor fields. These D7-branes fill $A d S_{5}$ and wrap the $S^{3} \subset S^{5}$. There are two remaining world volume scalars on the branes. One scalar is chosen to be trivially constant and the other a function of radial coordinate $\theta(u)$ which describes the position $S^{3}$ on the $S^{5}$ and is dual to the mass operator 23 .

The $U(1)$ worldvolume gauge field $A_{\mu}$ is dual to the $U(1)$ current $J^{\mu}$. We follow the conventions of [10] and turn on $A_{+}(y, u), A_{-}(y, u)$ and $A_{y}\left(x^{-}, u\right)$ as well as the $\theta(u)$. The above system is described by Dirac-Born-Infeld action as follows

$$
S_{D 7}=-N_{f} T_{D 7} \int e^{-\phi} d^{8} \xi \sqrt{-\operatorname{det}\left(g_{D 7}+\tilde{F}\right)},
$$

where $T_{D 7}, \xi, \tilde{F}=\left(2 \pi \alpha^{\prime}\right) F$ and $\phi$ are the D-brane tension, worldvolume coordinates, the normalized $U(1)$ field strength and the dilaton, respectively. The pullback of the spacetime metric with respect to the aforementioned embedding map is given by the metric $g_{D 7}$. We define $\mathcal{N}=2 \pi^{2} N_{f} T_{D 7}$ with $2 \pi^{2}$ from the integration of $S^{3} 2$.

From the gauge field equations of motion, conserved charges $I_{+}=\left\langle J^{+}\right\rangle, I_{-}=\left\langle J^{-}\right\rangle$ and $I_{y}=<J^{y}>$ associated with $A_{+}(y, u), A_{-}(y, u)$ and $A_{y}\left(x^{-}, u\right)$ are found. Following [10], we introduce the gauge filed $A_{\mu}$ in the light-cone coordinates as

$$
A_{+}=E_{b} y+h_{+}(u), \quad A_{-}=2 b^{2} E_{b} y+h_{-}(u), A_{y}=A_{y}(u), A_{u}=0 .
$$

where the electric field is redefined as $E_{b}=\frac{E}{2 b}$ to scale non-relativistically and it is the light-cone component of an electric field in the boost direction $\mathrm{x}$ [22]. Having worked out the gauge fields in terms of $I_{+}, I_{-}$and $E_{b}$, one finds the on-shell DBI action as follows

$$
S_{D 7}=-\tilde{\mathcal{N}}^{2} \int d u \hat{G}_{1}(u) \sqrt{G_{+-} g_{u u}^{D 7}} G_{y y}^{3 / 2} \frac{\xi}{\sqrt{\xi \chi-a}}
$$

where $g_{u u}^{D 7}=G_{u u}+\theta^{\prime}(u)$ and $\hat{G}_{1}(u)$ is related to the $S^{5}$ metric [10], also

$$
\begin{aligned}
& \xi=\tilde{E}_{b}^{2} G_{3}+G_{+-} G_{y y}, \quad \chi=G_{y y}\left(-I_{y}^{2}-\tilde{\mathcal{N}}^{2} \hat{G}_{1}(u) G_{y y} G_{+-}\right) \\
& a=G_{+-}\left[\tilde{E}_{b}^{2}\left(I_{+}+2 I_{-} b^{2}\right)^{2}+G_{y y}\left(I_{+}^{2} g_{++}+I_{-}\left(2 I_{+} g_{+-}+I_{-} g_{--}\right)\right)\right], \\
& G_{+-}=g_{++} g_{--} g_{+-}^{2}, \quad G_{3}=4 b^{4} g_{++}-4 b^{2} g_{+-}+g_{--},
\end{aligned}
$$

$\xi$, as a function of $u$, becomes zero at a specific point, $u_{c}$ where $\xi\left(u_{c}\right)=0$. This special point can be found by solving this equation

$$
\tilde{E}_{b}^{2} G_{3}\left(u_{c}\right)+G_{+-}\left(u_{c}\right) G_{y y}\left(u_{c}\right)=0 .
$$

\footnotetext{
${ }^{2}$ In $A d S_{5} \times S^{5}$ AdS black brane background, $e^{-\phi}$ is a constant and it can be absorbed in $\mathcal{N}$.
} 
Reality condition of $\sqrt{\xi \chi-a}$ imposes two functions $\chi$ and $a$ must vanish at $u=u_{c}$. Then one finds the non-relativistic DC conductivity as follows

$$
\sigma=2 \pi \alpha^{\prime} \sqrt{\frac{G_{3}\left(u_{c}\right)}{G_{y y}\left(u_{c}\right)}\left(\tilde{\mathcal{N}}^{2} G_{y y}\left(u_{c}\right)+\frac{I_{+}^{2}}{4 \tilde{E}_{b}^{2} b^{4}+g_{--}\left(u_{c}\right) G_{y y}\left(u_{c}\right)}\right)} .
$$

It is clearly seen that the non-relativistic conductivity has two main terms [22]. This is similar the relativistic case [23]. The term proportional to $I_{+}^{2}$ shows the contribution from the charge carriers. The other term describes the contribution from the charge-neutral terms which arises from thermally produced pairs of charge carriers.

Now we find the non-relativistic conductivity in the case of the AdS black brane where the metric functions are given by

$$
G_{t t}=-u^{2}\left(1-\frac{u_{0}^{4}}{u^{4}}\right), \quad G_{y y}=u^{2}, \quad G_{u u}=u^{-2}\left(1-\frac{u_{0}^{4}}{u^{4}}\right)^{-1}
$$

and the Hawking temperature in this frame is given by [10]

$$
T=\frac{u_{0}}{\pi b}
$$

Then (10) becomes 10

$$
\sigma(E, b, T)=2 \pi \alpha^{\prime} \sqrt{\frac{\tilde{\mathcal{N}} b^{2} \cos ^{6} \theta\left(u_{c}\right)}{16} \sqrt{4 \tilde{E}_{b}^{2}+\pi^{4} T^{4} b^{4}}+\frac{4 I_{+}^{2}}{4 \tilde{E}_{b}^{2}+\pi^{4} T^{4} b^{4}}},
$$

Then $\sigma$ depends on the new parameter $b$ which can be interpreted as a doping parameter [19]. In the next section, we more explain the importance of this parameter. It was pointed out in [10] that the expression of (13) is the analogues of the equation (3.27) of [22]. However, they are not the same except in some limiting cases 3 . If one neglects the charge-neutral term, for weak electric field compared to temperature (13) becomes

$$
\sigma_{1} \simeq \frac{4 \pi \alpha^{\prime} I_{+}}{\pi^{2} b^{2} T^{2}}
$$

For the opposite limit, one finds

$$
\sigma_{2} \simeq 2 \pi \alpha^{\prime} \frac{I_{+}}{b \tilde{E}_{b}}
$$

It is desirable to investigate (14) and (15) in the presence of higher derivative corrections. However, it is not our purpose to follow this calculation.

\section{Strange metals from AdS Light-Cone black hole}

In this section, we study the strange metal behavior in the ALCF. Then, one should choose the gauge fields as [19]

$$
A_{+}=E_{b} y+h_{+}(u), \quad A_{-}=2 b^{2} E_{b} y+h_{-}(u), \quad A_{y}=2 E_{b} b^{2} x^{-}+h_{y}(u) .
$$

\footnotetext{
${ }^{3}$ One may refer to [10] for more explanation of this point.
} 
The DBI action, then, reads

$$
S_{D 7}=-\mathcal{N} \int d^{5} \xi \mathcal{L}
$$

where the Lagranigian is given by 4

$$
\begin{aligned}
& \frac{\mathcal{L}^{2}}{\cos ^{6} \theta G_{y y}}=-\hat{G} G_{y y} G_{u u}-\tilde{E}_{b}^{2} \tilde{h}_{-}^{\prime 2}-\tilde{E}_{b}^{2} g_{--} G_{u u}- \\
& G_{y y}\left(g_{--} \tilde{h}_{+}^{\prime 2}-2 g_{+-} \tilde{h}_{+}^{\prime} \tilde{h}_{-}^{\prime}+g_{++} \tilde{h}_{-}^{\prime 2}\right)-\hat{G} \tilde{h}_{y}^{\prime 2} .
\end{aligned}
$$

Notice the tildes indicate that the quantities are scaled with the factor of $2 \pi \alpha^{\prime}$. Also

$$
\hat{G}=-g_{+-}^{2}+g_{--} g_{++} .
$$

The constants of motion are

$$
J=<J^{+}>=\frac{\partial \mathcal{L}}{\partial \tilde{h}_{+}^{\prime}}, \quad<J^{-}>=\frac{\partial \mathcal{L}}{\partial \tilde{h}_{-}^{\prime}}, \quad<J^{y}>=\frac{\partial \mathcal{L}}{\partial \tilde{h}_{y}^{\prime}} .
$$

Where $J,\left\langle J^{-}>\right.$and $\left\langle J^{y}>\right.$ are the light-cone charge density, light-cone current along $x_{-}$ direction and current along $y$ direction. The boundary value of the D7-brane worldvolume field $A_{+}$acts as a source for the field theory operator $J$. The bulk field $A_{-}$is dual to the field theory operator $J_{-}$. In the field theory, if one introduces $<J_{+}>$, one must also introduce $\left\langle J_{-}>[22\right.$.

Having worked out the gauge fields in terms of these constants one can write the on-shell Lagrangian as follows

$$
\begin{aligned}
\mathcal{L}=- & \cos ^{6} \theta G_{z z} \sqrt{\hat{G} g_{--} G_{y y} G_{z z} G_{u u}^{D 7}} \times \\
& \left(\frac{\left(\tilde{E}_{b}^{2} g_{--}-\hat{G} G_{y y}\right)^{2}}{\left(\tilde{E}_{b}^{2} g_{--}-\hat{G} G_{y y}\right) \chi-\hat{G} G_{y y} G_{z z}\left(g_{--}<J^{-}>+g_{+-}<J^{+}>\right)^{2}}\right)^{1 / 2},
\end{aligned}
$$

where $G_{u u}^{D 7}=G_{u u}+\theta^{\prime}(u)$ and

$$
\chi=g_{--} G_{y y}<J^{y}>^{2}-\hat{G}\left(g_{--} G_{y y} G_{z z} \cos ^{6} \theta+<J^{+}>^{2}\right) .
$$

We demand the big square root in the on-shell Lagrangian to be real all the way from the horizon to the boundary. The explicit form of the numerator depends on the background metric and it must be zero somewhere between the horizon and the boundary. We assign the value of $u$ where the numerator change the sign as $u_{*}$ and it can be found by solving this equation

$$
\left(\tilde{E}_{b}^{2} g_{--}+\hat{G} G_{y y}\right)_{u=u_{*}}=0 \text {. }
$$

The denominator should also vanish at $u=u_{*}$. By requiring this condition, one finds two important relations which imply that the light-cone charge density and light-cone current are not independent as

$$
<J^{-}>=\left(\frac{g_{+-}}{g_{--}}\right)_{u=u_{*}}<J^{+}>
$$

\footnotetext{
${ }^{4}$ We have assumed $G_{x x}=G_{y y}=G_{z z}$.
} 
, and Ohms law, $<J^{y}>=\sigma E_{b}$, with

$$
\begin{aligned}
\sigma^{2} & =\sigma^{(1)}+\sigma^{(2)} \\
& \sim \cos ^{6} \theta\left(\frac{g_{--}}{G_{y y}}\right)_{u=u_{*}}+J^{2}\left(\frac{1}{G_{y y}^{2}}\right)_{u=u_{*}} .
\end{aligned}
$$

Then the Ohmic DC conductivity is given by $\sigma$ which consists of two terms. The important behavior of strange metals can be found in the limit of large Light-Cone charge density $J$ [19]. We consider (25) in this limit and find that

$$
\rho \simeq 1 / \sigma^{(2)}=\frac{G_{y y}\left(u_{*}\right)}{J}
$$

Then one should find $G_{y y}\left(u_{*}\right)$ to investigate the temperature dependency of the resistivity. If one considers AdS-Schwarzschild black brane in the Light-Cone Frame, one finds that the Ohmic resistivity depends linearly on temperature [19]. We show this result in detail as follows.

The metric functions and the Hawking temperature are given in (11) and (12). As a result, (23) becomes

$$
-u^{6}+u^{2} u_{0}^{4}+\frac{b^{2} \tilde{E}_{b}^{2} u_{0}^{4}}{u^{2}}=0
$$

And $u_{*}$ is found as

$$
u_{*}^{2}=u_{0}\left(\frac{u_{0}^{2}+\sqrt{4 b^{2} \tilde{E}_{b}^{2}+u_{0}^{4}}}{2}\right)^{1 / 2}
$$

From the metric functions in (11), one finds that $G_{y y}\left(u_{*}\right)=u_{*}^{2}$. Then the resistivity without corrections (which is called $\rho^{(0)}$ )is given by

$$
\rho^{(0)}=\frac{\pi b T}{\sqrt{2} J} \sqrt{\pi^{2} b^{2} T^{2}+\sqrt{4 b^{2} \tilde{E}_{b}^{2}+\pi^{4} b^{4} T^{4}}}
$$

Now we consider low temperature regime of the model to study the leading behavior of the resistivity. In this regime one can change $b$ at fixed $\tilde{E}_{b}$ and $T$. For small $T$, one finds that

$$
\rho^{(0)}=\left(\frac{\pi b \sqrt{b \tilde{E}_{b}}}{J} T+\frac{\pi^{3} b^{3}}{4 \sqrt{b \tilde{E}_{b}}} T^{3}+a_{2 n+1}(b, \tilde{E}) T^{2 n+1}\right), \quad n=3,4,5, \ldots,
$$

Thus the first temperature dependence is linear. At small enough temperatures, by increasing the parameter $b$, one finds from (29) that a cross-over behavior of the resistivity from linear to quadratic in temperature exists and one finds another important property of the strange metals. It was argued in [19] that the crossover behavior is due to the fact that effectively the gravitational background interpolates between $\mathrm{z}=1$ (AdS) symmetry in the $\mathrm{UV}$ and $\mathrm{z}=2$ Lifshitz symmetry in the IR.

Now what will be happened if one considers $\mathcal{R}^{4}$ and $\mathcal{R}^{2}$ corrections to the AdS-Schwarzschild black brane? We call the resistivity in this case as $\rho^{(\lambda)}$ and $\rho^{\left(\lambda_{G B}\right)}$ and explore effect of the finite coupling corrections on this quantity in the next section. 


\subsection{The resistivity of strange metals at finite coupling}

Since $A d S / C F T$ correspondence refers to complete string theory, one should consider the string corrections to the 10D supergravity action. The first correction occurs at order $\left(\alpha^{\prime}\right)^{3}$ [24. In the extremal $A d S_{5} \times S^{5}$ it is clear that the metric does not change [25], conversely this is no longer true in the non-extremal case. Corrections in inverse 't Hooft coupling $1 / \lambda$ which correspond to $\alpha^{\prime}$ corrections on the string theory side were found in [24]. Functions of the $\alpha^{\prime}$-corrected metric are given by [26]

$$
\begin{aligned}
G_{t t} & =-u^{2}\left(1-w^{-4}\right) T(w), \\
G_{x x} & =u^{2} X(w), \\
G_{u u} & =u^{-2}\left(1-w^{-4}\right)^{-1} U(w),
\end{aligned}
$$

where

$$
\begin{aligned}
& T(w)=1-k\left(75 w^{-4}+\frac{1225}{16} w^{-8}-\frac{695}{16} w^{-12}\right)+\ldots \\
& X(w)=1-\frac{25 k}{16} w^{-8}\left(1+w^{-4}\right)+\ldots \\
& U(w)=1+k\left(75 w^{-4}+\frac{1175}{16} w^{-8}-\frac{4585}{16} w^{-12}\right)+\ldots,
\end{aligned}
$$

and $w=\frac{u}{u_{0}}$. There is an event horizon at $u=u_{0}$ and the geometry is asymptotically $A d S$ at large $u$ with a radius of curvature $R=1$. The expansion parameter $k$ can be expressed in terms of the inverse 't Hooft coupling as

$$
k=\frac{\zeta(3)}{8} \lambda^{-3 / 2} \sim 0.15 \lambda^{-3 / 2}
$$

The temperature in the light cone frame is given by

$$
T_{\mathcal{R}^{4}}=\frac{u_{0}}{b \pi(1-k)}
$$

By replacing (31) to (23), one finds the following equation

$$
\begin{aligned}
& 6950000 b^{32} k^{3} \pi^{32} T^{32}+6184375 b^{28} k^{3} \pi^{28} T^{28} u^{4}-125 b^{24} k^{2}(71168+67725 k) \pi^{24} T^{24} u^{8}+ \\
& 5 b^{16} k \pi^{16} T^{16}\left(569344 E^{2}+125 b^{4}(1584-11095 k) k \pi^{4} T^{4}\right) u^{12}+ \\
& 5 b^{12} k \pi^{12} T^{12}\left(-632064 E^{2}+b^{4}\left(569344+1973200 k+303125 k^{2}\right) \pi^{4} T^{4}\right) u^{16}+ \\
& 80 b^{12} k\left(-39664-12375 k+9375 k^{2}\right) \pi^{12} T^{12} u^{20}+ \\
& 16 b^{4} \pi^{4} T^{4}\left(16 E^{2}(16+1175 k)+25 b^{4}(16-2425 k) k \pi^{4} T^{4}\right) u^{24}+ \\
& 512 b^{4}(8+625 k) \pi^{4} T^{4} u^{28}-4096 u^{32}=0,
\end{aligned}
$$

which is a lengthy equation. Fortunately, we are going to take the small limit of temperature 5 , then we only keep terms to order $T^{8}$ and rewrite (35) as

$$
\begin{gathered}
256 u^{8}-32 T^{4} b^{4}(8+625 k) \pi^{4} u^{4}-16 T^{4} b^{6} \tilde{E}_{b}^{2}(16+1175 k)+ \\
25 b^{8} k(-16+2425 k) \pi^{8} T^{8}=0 .
\end{gathered}
$$

\footnotetext{
${ }^{5}$ We express the results in terms of the temperature of hot plasma without any corrections, $T_{\mathcal{R}^{4}}(1-k)=T$.
} 


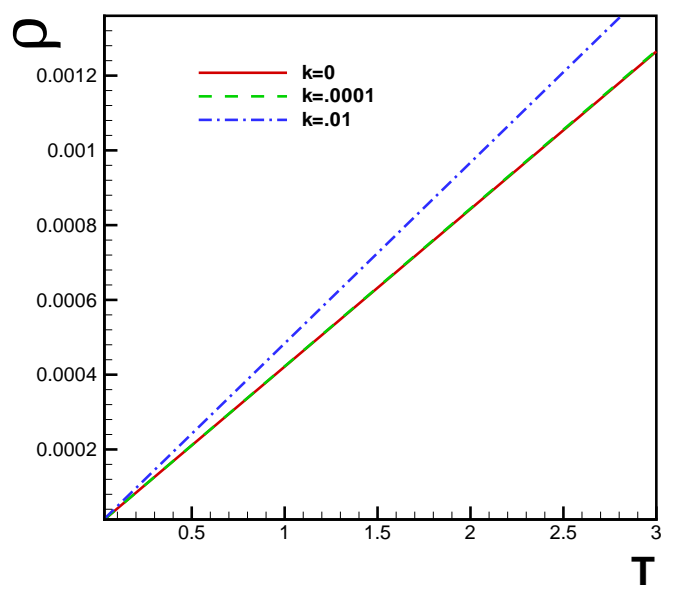

Figure 1: The resistivity of strange metals versus the temperature in the presence of $\mathcal{R}^{4}$ corrections. The different values of coupling constants are $k=0, k=0.0001, k=0.01$. Also $E=0.2$ and $b=0.0003$.

This equation should be solved to find $u_{*}$. It is clearly seen that (36) is the same as the equation of (27). Then one expects the same solution while the $\mathcal{R}^{4}$ corrections have been included $\sqrt{6}$. The final expansion of the resistivity at finite coupling as follows

$$
\rho^{(\lambda)}=\hat{a}_{1}(b, E, k) T+\hat{a}_{3}(b, E, k) T^{3}+\hat{a}_{2 n+1}(b, E, k) T^{2 n+1}, \quad n=0,1,2,3, \ldots
$$

where

$$
\begin{aligned}
& \hat{a}_{1}=\frac{1}{2}(16+1175 k)^{1 / 4}\left(\frac{\pi b \sqrt{b \tilde{E}_{b}}}{J}\right), \\
& \hat{a}_{3}=\frac{b^{3} \pi^{3}(8+575 k)}{16 J\left(b^{2} \tilde{E}_{b}^{2}(16+1175 k)\right)^{1 / 4}} .
\end{aligned}
$$

It can be seen that the result obtained in [19] can be reproduced even at higher-order corrections. This is an interesting result that $\mathcal{R}^{4}$ corrections keep the linear temperature dependent resistivity and at the leading order enhance the coefficient of it as

$$
\hat{a}_{1}=\frac{\pi b \sqrt{b \tilde{E}_{b}}}{2 J}\left(16+176.25 \lambda^{-3 / 2}\right)^{1 / 4} .
$$

To support our conclusion, we use the numerical methods and plot the resistivity in the presence of $\mathcal{R}^{4}$ corrections in Fig. 1. We assume $E=.2$ and $b=.0003$. For small values of $k$, for example $k=.0001$, the effect of corrections are negligible. It is clearly seen that by increasing the parameter $k$, the slope of line is changing.

Our numerical calculations also supports that by increasing the bulk parameter $b$, the temperature dependence of the resistivity crosses from linear to quadratic. This is another

\footnotetext{
${ }^{6}$ It should be noticed that we are interested in the case of small temperature regime.
} 
interesting property of strange metals which can be found from AdS Light-Cone black hole and is also valid in the presence of $\mathcal{R}^{4}$ corrections.

Next, we study $\mathcal{R}^{2}$ corrections to the resistivity and call it $\rho^{\left(\lambda_{G B}\right)}$. In five dimensions, we consider the theory of gravity with quadratic powers of curvature as Gauss-Bonnet(GB) theory. The exact solutions and thermodynamic properties of the black brane in GB gravity were discussed in [27, 28, 29]. The metric functions are given by

$$
G_{t t}=-N u^{2} h(u), \quad G_{u u}=\frac{1}{u^{2} h(u)}, \quad G_{x x}=G_{y y}=G_{z z}=u^{2},
$$

where

$$
h(u)=\frac{1}{2 \lambda_{G B}}\left[1-\sqrt{1-4 \lambda_{G B}\left(1-\frac{u_{0}^{4}}{u^{4}}\right)}\right] .
$$

In (40), $N=\frac{1}{2}\left(1+\sqrt{1-4 \lambda_{G B}}\right)$ is an arbitrary constant which specifies the speed of light of the boundary gauge theory and we choose it to be unity. The temperature is given by

$$
T_{G B}=\frac{\sqrt{N} u_{0}}{b \pi} .
$$

Then equation (23) reads

$$
\frac{u^{2}}{2 \lambda_{G B}}\left(2 b^{2} \lambda_{G B} \tilde{E}_{b}^{2}+N\left(u^{4}+b^{2} \tilde{E}_{b}^{2}\right)\left(-1+\sqrt{1+4 \lambda_{G B}\left(-1+\frac{u_{0}^{4}}{u^{4}}\right)}\right)\right)=0 .
$$

One should solve the above equation to find curvature-squared corrections to the Ohmic resistivity as follows 7

$$
\begin{aligned}
6 J \sqrt{\rho^{\lambda_{G B}}}= & \frac{b^{4} T^{4}}{N^{2}}-\frac{2(1-2 N) b^{2} \tilde{E}_{b}^{2}}{N}+ \\
& 22^{1 / 3}\left(b^{4} \tilde{E}_{b}^{4}\left(1-N+N^{2}-3 \lambda_{G B}\right)+\frac{2(1+N)}{N} b^{6} \tilde{E}_{b}^{2} T^{4}+\frac{b^{8} T^{8}}{N^{2}}\right) \times \\
& g\left(\tilde{E}_{b}, \lambda_{G B}, b\right)^{-1 / 3}+ \\
& \frac{22^{2 / 3}}{N^{2}}\left(I\left(\lambda_{G B}, E_{b}, b\right)+3 \sqrt{3} N^{3} b^{3} E_{b}^{3} \sqrt{h\left(\lambda_{G B}, E_{b}, b\right)}\right)^{1 / 3}
\end{aligned}
$$

\footnotetext{
${ }^{7}$ We also express here the results in terms of the temperature of hot plasma without any corrections, $\frac{T_{G B}}{\sqrt{N}}=T$.
} 
where

$$
\begin{aligned}
g\left(\tilde{E}_{b}, \lambda_{G B}, b\right)= & N^{3} b^{6} E_{b}^{6}\left(9 \lambda_{G B}-2\right)-2 N^{6}\left(b^{2} E_{B}^{2}+\frac{b^{4} T^{4}}{N^{2}}\right)^{3}+ \\
& 3 N^{4} b^{4} E_{b}^{4}\left(b^{2} E_{b}^{2}\left(1-6 \lambda_{G B}\right)+\left(3 \lambda_{G B}-2\right) \frac{b^{4} T^{4}}{N^{2}}\right)+ \\
& 3 N^{5}\left(b^{6} E_{b}^{6}-b^{4} E_{b}^{4}-\frac{2 b^{10} E_{b}^{2} T^{8}}{N^{4}}\right)+ \\
& 3 \sqrt{3} N^{3} b^{3} E_{b}^{3} \sqrt{h\left(\lambda_{G B}, E_{b}, b\right)}, \\
h\left(\lambda_{G B}, E_{b}, b\right)= & b^{6} E_{b}^{6} \lambda_{G B}^{2}\left(4 \lambda_{G B}-1\right)- \\
& 2 N b^{4} E_{b}^{4} \lambda_{G B}\left(b^{2} E_{b}^{2}\left(-1+4 \lambda_{G B}\right)+\frac{\lambda_{G B} b^{4} T^{4}}{N^{2}}\right)+ \\
& N^{4}\left(b^{2} E_{b}^{2}+\frac{B^{4} T^{4}}{N^{2}}\right)^{2}\left(b^{2} E_{b}^{2}\left(-1+4 \lambda_{G B}\right)+4 \frac{\lambda_{G B} b^{4} T^{4}}{N^{2}}\right)+ \\
& 2 N^{3} b^{2} E_{b}^{2}\left(b^{4} E_{b}^{4}\left(1-4 \lambda_{G B}\right)+\frac{b^{6} T^{4} E_{b}^{2}}{N^{2}}\left(-1+4 \lambda_{G B}\right)+5 \frac{\lambda_{G B} b^{8} T^{8}}{N^{4}}\right)+ \\
& N^{2}\left(b^{6} E_{b}^{6}\left(-1+2 \lambda_{G B}+8 \lambda_{G B}^{2}\right)+4 \frac{b^{8} T^{4} E_{b}^{4}}{N^{2}} \lambda_{G B}\left(2-5 \lambda_{G B}\right)-\frac{b^{10} T^{8} E_{b}^{2}}{N^{4}} \lambda_{G B}^{2}\right),
\end{aligned}
$$

and

$$
\begin{aligned}
I\left(\lambda_{G B}, E_{b}, b\right)= & N^{3} b^{6} E_{b}^{6}\left(9 \lambda_{G B}-2\right)-2 N^{6}\left(b^{2} E_{b}^{2}+\frac{b^{4} T^{4}}{N^{2}}\right)^{3}+ \\
& 3 N^{4} b^{4} E_{b}^{4}\left(b^{2} E_{b}^{2}\left(1-6 \lambda_{G B}\right)+\left(-2+3 \frac{\left.\lambda_{G B}\right) b^{4} T^{4}}{N^{2}}\right)+\right. \\
& 3 N^{5}\left(b^{6} E_{b}^{6}-b^{4} E_{b}^{8} T^{4}-\frac{2 b^{10} E_{b}^{2} T^{8}}{N^{4}}\right) .
\end{aligned}
$$

At the limit of large density and low temperature, one finds the following expansion

$$
\rho^{\left(\lambda_{G B}\right)}=A_{0}\left(b, \lambda_{G B}, E_{b}\right)+A_{4}\left(b, \lambda_{G B}, E_{b}\right) T^{4}+A_{8}\left(b, \lambda_{G B}, E_{b}\right) T^{8}+\ldots,
$$

It is clearly seen that the Ohmic resistivity can never be linear in the case of $\mathcal{R}^{2}$ corrections. This is an interesting result and one may define a new class of strange metals where the resistivity depends on $T^{4} 8$

Now, We increase the doping parameter $\mathrm{b}$ to study behavior of the resistivity at finite coupling. This limit was studied in details in [19. In this paper a dimensionless variable was defined as

$$
t=\frac{\pi R T b}{\sqrt{2 b \tilde{E}_{b}}} .
$$

Different limits of resistivity was studied as $t \gg 1$ and $t \ll 1$ which correspond to $b T \gg$ $\sqrt{2 b \tilde{E}_{b}}$ and $b T \ll \sqrt{2 b \tilde{E}_{b}}$, respectively. The parameters $b$ is an extra parameter which exists in ALCF geometry. It can be identified as doping parameter. Then by increasing $b$,

\footnotetext{
${ }^{8}$ Certainly, this class must be study more in details and other properties should be investigated.
} 
one may neglect the electric field and by increasing it the electric field becomes strong [20]. Now we follow these limits.

By increasing $b$, we neglect the electric field which means $\tilde{E}_{b} \rightarrow 0$. As a result (23) is simplified to $\hat{G} G_{y y}=0$. The specific solution of this equation occurs at the horizon $u_{*}=u_{0}$. For example in (27), by applying this limit one finds that $u_{*} \rightarrow u_{0}$. This limit is also valid in the presence of higher derivative corrections, i.e $\mathcal{R}^{4}$ and $\mathcal{R}^{2}$. This is another important property of strange metals that by increasing the doping parameter, one finds a cross-over from linear to quadratic in temperature. We find that higher derivative corrections to the resistivity keep this property and show the cross over to quadratic in temperature. This is an interesting result which shows that one of the important properties of strange metals

can be achieved by AdS Light-Cone approach even in the presence of higher derivative corrections.

\subsection{Hall conductivity of strange metals at finite coupling}

The inverse Hall angle is defined as follows

$$
\frac{\sigma^{y y}}{\sigma^{y z}}
$$

where $\sigma^{y y}$ and $\sigma^{y z}$ are Ohmic and Hall conductivity, respectively. The temperature dependence $\left(\sim T^{2}\right)$ of the inverse Hall angle is the typical property of the strange metal. To study the Hall conductivity of strange metals, one should turn on a magnetic field. We follow [19, 20] and introduce the gauge fields as

$$
\tilde{A}=\left(\tilde{E}_{b} y+h_{+}(u)\right) d x^{+}+h_{-}(u) d x^{-}+h_{y}(u) d y+\left(\tilde{B}_{b} y\right) d z .
$$

It implies that there is an electric field, $E_{b}$, along the $y$ direction and a magnetic field, $B_{b}$, along the $z$ direction. The on-shell DBI action is

$$
S_{D 7}=-\tilde{\mathcal{N}}^{2} \int d u \sqrt{G_{+-} g_{--} g_{u u}^{D 7}} G_{y y} \frac{\xi_{1}}{\sqrt{\xi_{1} \chi_{1}-G_{y y}^{2} G_{+-} a_{1}^{2}-a_{2}^{2}}},
$$

where $g_{u u}^{D 7}=G_{u u}+\theta^{\prime}(u)$ and $\hat{G}_{i}(u)$ is related to the $S^{5}$ metric [10], also

$$
\begin{aligned}
& \xi_{1}=\tilde{E}_{b}^{2} G_{y y} g_{--}-G_{+-}\left(G_{y y}^{2}+\tilde{B}_{b}^{2}\right), \\
& \chi_{1}=-G_{+-}\left(g_{--} G_{y y}^{2} \cos (\theta)^{3}+I_{+}^{2}\right)+g_{--} G_{y y}\left(I_{z}^{2}+I_{y}^{2}\right), \\
& a_{1}=g_{--} I_{-}+g_{+-} I_{+}, \quad a_{2}=g_{--} G_{y y} \tilde{E}_{b} I_{z}+G_{+-} \tilde{B}_{b} I_{+}, \\
& G_{+-}=g_{++} g_{--} g_{+-}^{2},
\end{aligned}
$$

Reality condition imposes that $\xi_{1}\left(u_{c}\right)=0$. Then one should solve the following equation

$$
\tilde{E}_{b}^{2} G_{y y} g_{--}-G_{+-}\left(G_{y y}^{2}+\tilde{B}_{b}^{2}\right)=0,
$$

The Ohmic conductivity in the presence of magnetic field is given by

$$
\sigma^{y y}=\frac{\sqrt{G_{y y} g_{--} \cos (\theta)^{3}\left(G_{y y}^{2}+\tilde{B}_{b}^{2}\right)+I_{+}^{2}}}{G_{y y}^{2}+\tilde{B}_{b}^{2}},
$$


and the Hall conductivity is

$$
\sigma^{y z}=\frac{\tilde{B}_{b} I_{+}}{G_{y y}^{2}+\tilde{B}_{b}^{2}}
$$

These conductivities must be evaluated at $u=u_{c}$ which can be found from (52). At large density and weak magnetic field, one finds that

$$
\sigma^{y y} \sim \frac{I_{+}}{G_{y y}\left(u_{c}\right)}, \quad \sigma^{y z}=\frac{\tilde{B}_{b} I_{+}}{G_{y y}\left(u_{c}\right)^{2}},
$$

As a result, the inverse Hall conductivity is given by the following expression

$$
\frac{\sigma^{y y}}{\sigma^{y z}} \sim \frac{G_{y y}\left(u_{c}\right)}{\tilde{B}_{b}}
$$

Then simply one should find $G_{y y}\left(u_{c}\right)$. To investigate the strange metal behavior, one should consider special limits of magnetic filed, electric field and temperature. Fortunately, ALCF benefits from extra parameter $b$. To more clarify the role of this parameter, we solve (52) in the case of AdS spacetime withought any corrections. This case was studied in [19, 20]. From the metric functions in (11), one finds

$$
-u^{8}+\tilde{E}_{b}^{2} b^{6} T^{4}+u^{4} b^{4} T^{4}+\tilde{B}_{b}^{2}\left(b^{4} T^{4}-u^{4}\right)=0 .
$$

The analytic solution of the above equation would be easily found. However, we are going to understand different limits of parameters in this equation and apply it in the case of complicated equation which appears in higher derivative corrections. We consider different limits as follows

- $\tilde{B}_{b}=0$, by increasing the doping parameter $\left(\tilde{E}_{b} \rightarrow 0\right)$, one may neglect the electric field. In this case $u_{c}=b T$ then $G_{y y}\left(u_{c}\right)=u_{c}^{2} \sim T^{2}$

- $\tilde{B}_{b}=0$, by decreasing the doping parameter $\left(\tilde{E}_{b} \rightarrow \infty\right)$, one may keep only the term which depends on the electric field. In this case $u_{c} \sim T$ then $G_{y y}\left(u_{c}\right)=u_{c}^{2} \sim T$

- $\tilde{B}_{b} \neq 0$, one may assume $\tilde{B}_{b}>b \tilde{E}_{b}$ and decrease the doping parameter. In this case, one should only keep the terms which are depended on the magnetic field and (57) becomes $\tilde{B}_{b}^{2}\left(b^{4} T^{4}-u^{4}\right)=0$. Then $u_{c}=b T$ and $G_{y y}\left(u_{c}\right) \sim T^{2}$.

Now, one finds that in the presence of higher derivative corrections, the behavior of the inverse Hall effect will not be changed. Because in the limit of nonezero magnetic field and $\tilde{B}>\tilde{E}$ by decreasing the doping parameter, (52) becomes

$$
-G_{+-} \tilde{B}_{b}^{2}=0 \text {. }
$$

Where simply the solution is $u_{c}=u_{0}$.

\section{Conclusion}

It is well known that the light cone quantization of a relativistic theory looks like a nonrelativistic theory. As a simple holographic system, one may use the AdS-Schwarzshild 
black hole in light-con coordinates to investigate strange metal properties in (1). The universal experimental results of strange metals were studied in [19]. The non-relativistic DC conductivity is particularly important which is characterized by

$$
\sigma \sim \frac{1}{T}
$$

As it is clear the resistance $\rho=\frac{1}{\sigma}$ increases linearly with the temperature, this is the reason why 'strange' is defined [4]. One should notice that (59) is valid at low temperatures compared to the mass and density. In this paper, we have studied the effects of finite but large couplings by adding higher-derivative corrections in the gravity background. Especially, $\mathcal{R}^{4}$ terms and Gauss-Bonnet gravity has been considered. It is found that the Ohmic resistivity and inverse Hall angle are linear and quadratic temperature dependent in the case of $\mathcal{R}^{4}$ corrections, respectively. While Ohmic conductivity can never be linear temperature dependent and $\rho=A_{0}+A_{4} T^{4}+A_{8} T^{8}$. 9 This is an interesting result and may be define a new class of strange metals.

\section{Acknowledgment}

We would like to thank M. Ali-Akbari, M. Alishahiha and M. Sheikh-Jabbari for very useful discussions and especially thank Bom Soo Kim for reading the manuscript and useful comments. This research was supported by Shahrood University of Technology.

\section{References}

[1] S. A. Hartnoll, Lectures on holographic methods for condensed matter physics, Class.Quant.Grav. 26 (2009) 224002, arXiv:0903.3246].

J. McGreevy, Holographic duality with a view toward many-body physics, Adv.High Energy Phys. 2010 (2010) 723105, arXiv:0909.0518.

S. A. Hartnoll, Horizons, holography and condensed matter, arXiv:1106.4324.

N. Iqbal, H. Liu and M. Mezei, "Lectures on holographic non-Fermi liquids and quantum phase transitions," arXiv:1110.3814 [hep-th].

[2] G. R. Stewart, Non-Fermi-liquid behavior in d- and f-electron metals, Rev. Mod. Phys. 73, 797 (2001) [Addendum-ibid. 78, 743 (2006)].

[3] R. A. Cooper, Y. Wang, B. Vignolle, O. J. Lipscombe, S. M. Hayden, Y. Tanabe, T. Adachi, Y. Koike, M. Nohara, H. Takagi, C. Proust, and N. E. Hussey, Anomalous criticality in the electrical resistivity of La2-xSrxCuO4, Science, 323, 603 (2009).

[4] S. Sachdev, "Strange metals and the AdS/CFT correspondence," J. Stat. Mech. 1011 (2010) P11022 arXiv:1010.0682 [cond-mat.str-el]].

[5] D. T. Son, "Toward an AdS/cold atoms correspondence: A Geometric realization of the Schrodinger symmetry," Phys. Rev. D 78 (2008) 046003 [arXiv:0804.3972 [hep-th]].

[6] K. Balasubramanian and J. McGreevy, "Gravity duals for non-relativistic CFTs," Phys. Rev. Lett. 101 (2008) 061601 [arXiv:0804.4053 [hep-th]].

\footnotetext{
${ }^{9} A_{0}, A_{4}$ and $A_{8}$ depend on the electric field, Gauss-Bonnet coupling and parameter $b$.
} 
[7] J. Maldacena, D. Martelli and Y. Tachikawa, "Comments on string theory backgrounds with non-relativistic conformal symmetry," JHEP 0810 (2008) 072 arXiv:0807.1100 [hep-th]].

[8] M. Alishahiha and O. J. Ganor, "Twisted backgrounds, PP waves and nonlocal field theories," JHEP 0303 (2003) 006 arXiv:hep-th/0301080.

[9] W. D. Goldberger, "AdS/CFT duality for non-relativistic field theory," JHEP 0903 (2009) 069 arXiv:0806.2867 [hep-th]].

J. L. F. Barbon and C. A. Fuertes, "On the spectrum of nonrelativistic AdS/CFT," JHEP 0809 (2008) 030 arXiv:0806.3244 [hep-th]].

[10] B. S. Kim and D. Yamada, "Properties of Schroedinger Black Holes from AdS Space," JHEP 1107 (2011) 120 arXiv:1008.3286 [hep-th]].

[11] T. Faulkner, N. Iqbal, H. Liu, J. McGreevy and D. Vegh, "Strange metal transport realized by gauge/gravity duality," Science 329 (2010) 1043.

[12] C. Charmousis, B. Gouteraux, B. S. Kim, E. Kiritsis and R. Meyer, "Effective Holographic Theories for low-temperature condensed matter systems," JHEP 1011 (2010) 151 [arXiv:1005.4690 [hep-th]].

[13] R. C. Myers, S. Sachdev and A. Singh, "Holographic Quantum Critical Transport without Self-Duality," Phys. Rev. D 83 (2011) 066017 [arXiv:1010.0443 [hep-th]].

[14] S. S. Pal, "Model building in AdS/CMT: DC Conductivity and Hall angle," Phys. Rev. D 84 (2011) 126009 [arXiv:1011.3117 [hep-th]].

[15] B. -H. Lee, D. -W. Pang and C. Park, "Strange Metallic Behavior in Anisotropic Background," JHEP 1007 (2010) 057 [arXiv:1006.1719 [hep-th]].

[16] R. Meyer, B. Gouteraux and B. S. Kim, "Strange Metallic Behaviour and the Thermodynamics of Charged Dilatonic Black Holes," Fortsch. Phys. 59 (2011) 741 arXiv:1102.4433 [hep-th]].

[17] B. -H. Lee and D. -W. Pang, "Notes on Properties of Holographic Strange Metals," Phys. Rev. D 82 (2010) 104011 arXiv:1006.4915 [hep-th]].

[18] S. A. Hartnoll, J. Polchinski, E. Silverstein and D. Tong, "Towards strange Metallic holography,"

[19] B. S. Kim, E. Kiritsis and C. Panagopoulos, "Holographic quantum criticality and strange metal transport," New J. Phys. 14 (2012) 043045 arXiv:1012.3464 [condmat.str-el]].

[20] K. -Y. Kim, D. -W. Pang, "Holographic DC conductivities from the open string metric," JHEP 1109 (2011) 051. arXiv:1108.3791 [hep-th]].

[21] M. Ali-Akbari and K. B. Fadafan, "Conductivity at finite 't Hooft coupling from AdS/CFT," arXiv:1008.2430 [hep-th]. 
[22] M. Ammon, C. Hoyos, A. O'Bannon and J. M. S. Wu, "Holographic Flavor Transport in Schrodinger Spacetime," JHEP 1006 (2010) 012 [arXiv:1003.5913 [hep-th]].

[23] A. Karch and A. O'Bannon, 'Metallic AdS/CFT," JHEP 0709 (2007) 024 arXiv:0705.3870 [hep-th]].

[24] J. Pawelczyk and S. Theisen, $A d S_{5} \times S^{5}$ black hole metric at $O\left(\alpha^{\prime 3}\right)$, JHEP $9809(1998)$ 010, hep-th/9808126;

[25] T. Banks and M. B. Green, "Non-perturbative effects in AdS(5) x $\mathrm{S}^{* *} 5$ string theory and d = 4 SUSY Yang-Mills," JHEP 9805, 002 (1998) arXiv:hep-th/9804170;

[26] S.S. Gubser, I.R. Klebanov and A.A. Tseytlin, Coupling constant dependence in the thermodynamics of $N=4$ supersymmetric Yang-Mills theory Nucl. Phys. B534 (1998) 202, hep-th/9805156;

[27] R. G. Cai, "Gauss-Bonnet black holes in AdS spaces," Phys. Rev. D 65 (2002) 084014 arXiv:hep-th/0109133.

[28] S. Nojiri and S. D. Odintsov, "Anti-de Sitter black hole thermodynamics in higher derivative gravity and new confining-deconfining phases in dual CFT," Phys. Lett. B 521 (2001) 87 [Erratum-ibid. B 542 (2002) 301] arXiv:hep-th/0109122.

[29] S. Nojiri and S. D. Odintsov, "(Anti-) de Sitter black holes in higher derivative gravity and dual conformal field theories," Phys. Rev. D 66 (2002) 044012 arXiv:hep-th/0204112. 